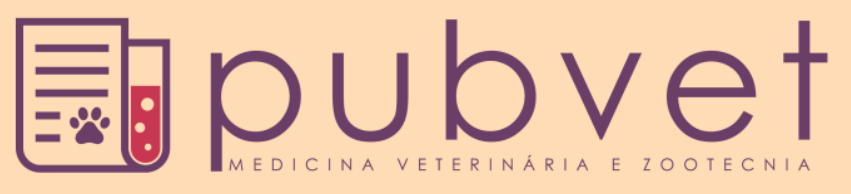

HTTP://DX.DOI.ORG/10.22256/PUBVET.V11N10.1046-1056

\title{
Resposta de pastagens de Trachypogon plumosus consorciadas com Stylosanthes capitata cv. Lavradeiro a níveis de fósforo e potássio
}

\author{
Newton de Lucena Costa ${ }^{1 *}$, Anibal de Moraes $^{2}$, Paulo César Faccio de Carvalho ${ }^{3}$, Vicente \\ Gianluppi $^{4}$, João Avelar Magalhães ${ }^{5}$ \\ ${ }^{1}$ Eng. Agr., D.Sc., Embrapa Roraima, Boa Vista, RR. \\ ${ }^{2}$ Professor Adjunto, Departamento de Fitotecnia e Fitossanitarismo da UFPR, Curitiba, PR. \\ ${ }^{3}$ Professor Adjunto, Departamento de Plantas Forrageiras e Agrometeorologia da UFRGS, Porto Alegre, RS \\ ${ }^{4}$ Eng. Agr., M.Sc., Embrapa Roraima, Boa Vista, RR. \\ ${ }^{5}$ Med. Veterinário, D.Sc., Embrapa Meio Norte, Parnaíba, PI. \\ *Autor para correspondência, E-mail: newton.lucena-costa@embrapa.br
}

\begin{abstract}
RESUMO. Avaliou-se o efeito da adubação fosfatada (0, 40, 80 e $120 \mathrm{~kg}$ de $\left.\mathrm{P}_{2} \mathrm{O}_{5} \mathrm{ha}^{-1}\right)$ e potássica $\left(0,40,80\right.$ e $\left.120 \mathrm{~kg}_{\text {de }} \mathrm{K}_{2} \mathrm{O} \mathrm{ha}{ }^{-1}\right)$ sobre a produtividade de forragem, composição química e extração de macronutrientes por pastagens nativas de Trachypogon plumosus consorciadas com Stylosanthes capitata cv. Lavradeiro nos cerrados de Roraima. Os efeitos da adubação fosfatada e potássica sobre a produtividade de forragem da leguminosa foram positivos e lineares, enquanto que para a gramínea e a soma dos componentes (gramínea + leguminosa) foram quadráticos e os máximos rendimentos estimados com a aplicação de

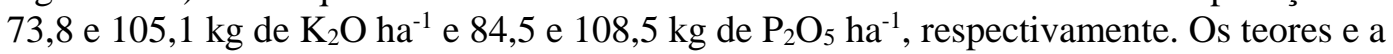
extração de nitrogênio, fósforo, cálcio, magnésio e potássio pela gramínea e leguminosa foram quadraticamente afetados pela adubação fosfatada e potássica. As extrações dos macronutrientes pela gramínea e leguminosa, independentemente da adubação fosfatada ou potássica, foram maiores para $\mathrm{N}$ e $\mathrm{K}$, seguidas de $\mathrm{Ca}, \mathrm{Mg}$ e $\mathrm{P}$. A adubação fosfatada e potássica proporcionaram forragem da gramínea e leguminosa com melhor qualidade e menores teores de fibra. Considerando-se a disponibilidade total de forragem e a composição botânica da pastagem - relação gramínea/leguminosa satisfatória - a utilização de doses de entre 80 e $90 \mathrm{~kg}$ de $\mathrm{P}_{2} \mathrm{O}_{5} \mathrm{ha}^{-1}$ e 60 e $70 \mathrm{~kg}$ de $\mathrm{K}_{2} \mathrm{O}$ ha- ${ }^{-1}$ podem assegurar o estabelecimento, a estabilidade produtiva, o fornecimento de forragem de boa qualidade $\mathrm{e}$ a persistência da consorciação.
\end{abstract}

Palavras chave: Cálcio, composição botânica, fibra, fósforo, magnésio, matéria seca, nitrogênio, potássio

\section{Response of Trachypogon plumosus pastures intercropping with Stylosanthes capitata cv. Lavradeiro to phosphate and potassium levels}

ABSTRACT. Was evaluated the effect of phosphate $\left(0,40,80\right.$ and $120 \mathrm{~kg}$ of $\left.\mathrm{P}_{2} \mathrm{O}_{5} \mathrm{ha}^{-1}\right)$ and potassic fertilization $\left(0,40,80\right.$ and $\left.120 \mathrm{~kg}_{\text {of }} \mathrm{K}_{2} \mathrm{O} \mathrm{ha}^{-1}\right)$ on the forage yield, chemical composition and macronutrients extraction of native pastures of Trachypogon plumosus intercropping with Stylosanthes capitata cv. Lavradeiro in Roraima's savannas. The effects of phosphate and potassic fertilization on legume forage yield were positive and linear, while for the grass and sum of components (grass + legume) were quadratic and the maximum yields estimated with the application of 73.8 and $105.1 \mathrm{~kg} \mathrm{of} \mathrm{K}_{2} \mathrm{O} \mathrm{ha}^{-1}$ and 84.5 and $108.5 \mathrm{~kg}$ of $\mathrm{P}_{2} \mathrm{O}_{5} \mathrm{ha}^{-1}$, respectively. Nitrogen, phosphorus, calcium, magnesium and potassium contents and extraction of grass and legume were quadratically affected by 
phosphate and potassic fertilization. Macronutrient extractions by grass and legume, independently of phosphate and potassic fertilization, were higher for $\mathrm{N}$ and $\mathrm{K}$, followed by $\mathrm{Ca}, \mathrm{Mg}$ and $\mathrm{P}$. Phosphate and potassic fertilization provided grass and legume forage with better quality and lower fiber contents. Considering the overall availability of forage and pasture botanical composition - satisfactory grass/legume relationship - the use of phosphate levels between 80 and $90 \mathrm{~kg}$ of $\mathrm{P}_{2} \mathrm{O}_{5}$ ha $^{-1}$ and 60 and $70 \mathrm{~kg} \mathrm{de} \mathrm{K}_{2} \mathrm{O}$ ha ${ }^{-1}$ can allow the establishment and yield stability and persistence of grass-legume mixture.

Keywords: Botanic composition, calcium, dry matter, fiber, nitrogen, phosphorus, potassium, dry matter

\title{
Respuesta de pastos de Trachypogon plumosus consorciados con Stylosanthes capitata cv. Lavradeiro a niveles de fósforo y potasio
}

\begin{abstract}
RESUMEN. Se evaluó el efecto de la fertilización fosfatada $\left(0,40,80\right.$ y $120 \mathrm{~kg}$ de $\mathrm{P}_{2} \mathrm{O}_{5}$ $\left.\mathrm{ha}^{-1}\right)$ y potásica $\left(0,40,80\right.$ y $120 \mathrm{~kg}$ de $\left.\mathrm{K}_{2} \mathrm{O} \mathrm{ha}{ }^{-1}\right)$ sobre la productividad de forraje, composición química y extracción de macronutrientes por pastos nativos de Trachypogon plumosus em mezcla con Stylosanthes capitata cv. Lavadeiro en las sabanas de Roraima. Los efectos de la fertilización fosfatada y potásica sobre la productividad de forraje de la leguminosa fueron positivos y lineales, mientras que para la gramínea y la suma de los componentes (gramínea + leguminosa) fueron cuadráticos y los máximos rendimientos estimados con la aplicación de 73,8 y 105,1 kg de $\mathrm{K}_{2} \mathrm{O} \mathrm{ha}^{-1}$ y 84,5 y $108,5 \mathrm{~kg} \mathrm{de}_{2} \mathrm{O}_{5}$ ha $^{-1}$, respectivamente. Los contenidos y la extracción de nitrógeno, fosforo, calcio, magnesio y potasio por la gramínea y leguminosa fueron cuadraticamente afectados por la fertilización fosfatada y potásica. Las extracciones de los macronutrientes por la gramínea y leguminosa, independientemente de la fertilización fosfatada o potásica, fueron mayores para $\mathrm{N} \mathrm{y} \mathrm{K}$, seguidas de $\mathrm{Ca}, \mathrm{Mg}$ y $\mathrm{P}$. La fertilización fosfatada y potásica proporcionó forraje de la gramínea y leguminosa con mejor calidad y menores contenidos de fibra. Considerando la disponibilidad total del forraje y la composición botánica de la pastura - relación gramínea/leguminosa satisfactoria - la utilización de dosis entre 80 y $90 \mathrm{~kg} \mathrm{de} \mathrm{P}_{2} \mathrm{O}_{5} \mathrm{ha}^{-1}$ y 60 y $70 \mathrm{~kg}$ de $\mathrm{K}_{2} \mathrm{O}$ ha $^{-1}$ pueden asegurar el establecimiento, la estabilidad productiva, el suministro de forraje de buena calidad y la persistencia de la consorciación.
\end{abstract}

Palabras clave: Calcio, composición botánica, fibra, fósforo, magnesio, materia seca, nitrógeno, potasio

\section{Introdução}

Nos cerrados de Roraima, as pastagens nativas representam importante recurso forrageiro para a alimentação dos ruminantes. Apesar das limitações quantitativas e qualitativas, decorrentes da baixa fertilidade natural dos solos, historicamente, as pastagens nativas proporcionaram o suporte alimentar para a exploração pecuária, que ao longo dos anos, tornou-se uma das principais atividades econômica de Roraima (Costa et al., 2009). O pastoreio contínuo com taxa de lotação variável, mas em geral extensivo e desvinculado do ritmo estacional de crescimento das pastagens e a não reposição dos nutrientes extraídos constituem as principais causas do esgotamento da fertilidade do solo do ecossistema e, consequentemente, da redução da capacidade de suporte e do potencial produtivo das pastagens nativas ao longo dos anos
(Sarmiento, 1992, Sarmiento et al., 2006, Ries and Shugart, 2008). Nas áreas planas e não inundáveis dos cerrados, predomina a gramínea Trachypogon plumosus (Humb. \& Bonpl. Ex Willd.) Nees, representando entre 80 e $90 \%$ da composição botânica das pastagens nativas, contudo a produção animal é muito baixa e necessário entre 4 e 8 ha para a manutenção de um bovino adulto, o que pode inviabilizar economicamente a atividade pecuária, desde que não sejam implementadas práticas de manejo adequadas para o seu melhoramento (Costa et al., 2009).

Para a obtenção de níveis satisfatórios de produção de forragem e, consequentemente do desempenho animal, torna-se necessário a utilização de alguma fonte de nitrogênio $(\mathrm{N})$, química ou biológica, já que sua baixa disponibilidade é uma das principais causas da baixa produtividade biológica primária das 
pastagens nativas (Townsend et al., 2012). A deficiência de $\mathrm{N}$ reflete a diminuição dos teores de matéria orgânica do solo, como consequência do uso frequente das queimadas como prática usual de manejo visando à eliminação da forragem não consumida e senescida (Moreira and Arruda, 1990). Face aos altos custos dos fertilizantes nitrogenados, a introdução de leguminosas em pastagens nativas, vem sendo recomendada como a alternativa mais eficiente e econômica para o fornecimento de $\mathrm{N}$ ao sistema solo-planta, além de aumentar a capacidade de suporte, prolongar o período de pastejo e melhorar o valor nutritivo da forragem em oferta (Moreira, 2006, Costa et al., 2013b). Dentre as várias leguminosas forrageiras introduzidas e avaliadas em Roraima, Stylosanthes capitata cv. Lavradeiro destacou-se entre as mais promissoras, pois, além de sua excelente produtividade de forragem, bom valor nutritivo e grande capacidade de colonização do solo, apresenta boa adaptação a solos de baixa fertilidade natural, alta tolerância à seca e excelente regeneração natural, em decorrência da produção de grandes quantidades de sementes (Gianluppi et al., 2001, Costa et al., 2009).

Em Roraima, a baixa disponibilidade de fósforo $(\mathrm{P})$ e potássio $(\mathrm{K})$, na maioria de seus solos, foi considerada como os fatores mais limitantes ao estabelecimento de pastagens consorciadas, reduzindo significativamente os rendimentos e a qualidade da forragem, além de sua persistência (Costa et al., 2009). O P desempenha importante papel no desenvolvimento do sistema radicular e no perfilhamento das gramíneas e na maximização dos processos de fixação biológica de nitrogênio das leguminosas, sendo indispensável à fotossíntese, síntese e degradação dos carboidratos, além de participar ativamente da respiração celular, influenciando o armazenamento, transporte e utilização da energia produzida no processo fotossintético (Fabrice et al., 2015). O K tem ação fundamental no metabolismo vegetal, notadamente no processo de fotossíntese, atuando nas reações de transformação da energia luminosa em química, além de participar na síntese de proteínas; neutralização de ácidos orgânicos e na regulação da pressão osmótica e do $\mathrm{pH}$ interno da planta; uso mais eficiente da água, através do melhor controle na abertura e fechamento dos estômatos (Costa, 2004) Nabinger and Carvalho (2009) recomendam a aplicação de níveis moderados de fertilizantes em pastagens nativas, de modo a favorecer a manutenção e produtividade das espécies de maior interesse forrageiro, evitando o aparecimento e proliferação de espécies oportunistas, beneficiadas momentaneamente pela melhoria do ambiente de produção e que não apresentam, no longo prazo, adaptação às condições edafoclimáticas do ecossistema pastoril.

Neste trabalho foram avaliados os efeitos da adubação fosfatada e potássica sobre a produção de forragem, composição química e extração de macronutrientes por pastagens de Trachyogon plumosus consorciadas com Stylosanthes capitata cv. Lavradeiro, nos cerrados de Roraima.

\section{Material e Métodos}

O ensaio foi conduzido no Campo Experimental da Embrapa Roraima, localizado em Boa Vista, durante o período de junho de 2013 a abril de 2015, em uma pastagem nativa em que a gramínea Trachypogon plumosus representava 95\% de sua composição botânica. O clima da região, segundo a classificação de Köppen and Geiger (1928) é Awi, caracterizado por períodos seco e chuvoso bem definidos, com aproximadamente seis meses cada um. A precipitação média anual é de $1.600 \mathrm{~mm}$ e $80 \%$ ocorrem nos seis meses do período chuvoso (abril a setembro). O solo da área experimental é um Latossolo Amarelo, textura média, com as seguintes características químicas, na profundidade de $0-20 \mathrm{~cm}: \mathrm{pH}_{\mathrm{H} 2 \mathrm{O}}=4,8 ; \mathrm{P}=1,9$ $\mathrm{mg} / \mathrm{kg} ; \mathrm{Ca}+\mathrm{Mg}=0,953 \mathrm{cmol}_{\mathrm{c}} \cdot \mathrm{dm}^{-3} ; \mathrm{K}=0,011$ $\mathrm{cmol}_{\mathrm{c}} \cdot \mathrm{dm}^{-3} ; \mathrm{Al}=0,61 \mathrm{cmol}_{\mathrm{c}} \mathrm{dm}^{-3} ; \mathrm{H}+\mathrm{Al}=2,64$ $\mathrm{cmol}_{\mathrm{c}} \cdot \mathrm{dm}^{-3}$ e Soma de Bases $=0,964 \mathrm{cmol}_{\mathrm{c}} \cdot \mathrm{dm}^{-3}$.

$\mathrm{O}$ delineamento experimental foi em blocos ao acaso com parcelas divididas e três repetições. As parcelas principais consistiram de quatro níveis de fósforo $\left(0,40,80\right.$ e $120 \mathrm{~kg}$ de $\left.\mathrm{P}_{2} \mathrm{O}_{5} \mathrm{ha}^{-1}\right)$ e as subparcelas de quatro níveis de potássio $(0,40,80$ e $120 \mathrm{~kg} \mathrm{de}_{2} \mathrm{O} \mathrm{ha}{ }^{-1}$ ), aplicados sob a forma de superfosfato triplo e cloreto de potássio, respectivamente. A leguminosa utilizada foi o Stylosanthes capitata cv. Lavradeiro, semeada a lanço e sem nenhum revolvimento do solo, após o rebaixamento da pastagem a $10 \mathrm{~cm}$ acima do solo, utilizando-se densidade de semeadura de $3,0 \mathrm{~kg}$ $\mathrm{ha}^{-1}$ de sementes (Valor cultural $=100 \%$ ). O tamanho das parcelas foi de 3,0 x 3,0 m, sendo a área útil de $4,0 \mathrm{~m}^{2}$. Durante $\mathrm{o}$ período experimental foram realizados onze cortes a intervalos de 56 dias.

Os rendimentos de forragem da gramínea e da leguminosa foram estimados através de cortes 
mecânicos, realizados a uma altura de $20,0 \mathrm{~cm}$ acima do solo. Em cada avaliação, após a separação dos componentes da consorciação (gramínea e leguminosa), o material colhido foi acondicionado em sacos de papel e pesado para estimativa da produção de biomassa verde e, posteriormente, colocado para secar em estufa de ventilação forçada a $65^{\circ} \mathrm{C}$ por 72 horas para determinação da produção de matéria seca verde (MSV) e moagem em peneira com malha de 5,0 $\mathrm{mm}$. Os teores de nitrogênio $(\mathrm{N})$ foram analisados de acordo com procedimentos descritos por Silva and Queiroz (2002); enquanto que os teores de fósforo $(\mathrm{P})$, cálcio $(\mathrm{Ca})$, magnésio $(\mathrm{Mg})$ e potássio (K) foram determinados conforme a metodologia descrita por Silva (2009). Os teores de P e K foram quantificados após digestão nitroperclórica. O P foi determinado por colorimetria; o $\mathrm{K}$ por fotometria de chama e $\mathrm{Ca}$ e $\mathrm{Mg}$ por espectrofotometria de absorção atômica. Os teores de fibra em detergente neutro (FDN) e fibra em detergente ácido (FDA) foram determinados pela metodologia proposta por Van Soest et al. (1991). A extração dos nutrientes foi estimada multiplicando-se os teores de cada nutriente pela quantidade de forragem acumulada em cada avaliação.

Os dados foram submetidos à análise de variância e de regressão considerando o nível de significância de 5\% de probabilidade, utilizandose o programa Sisvar (Ferreira, 2011). Para se estimar a resposta dos parâmetros avaliados, em função dos níveis de adubação fosfatada, a escolha dos modelos de regressão baseou-se na significância dos coeficientes linear e quadrático, por meio do teste " $\mathrm{t}$ ", de Student, ao nível de 5\% de probabilidade.

\section{Resultados e Discussão}

Os rendimentos de MSV da gramínea, leguminosa e soma dos componentes (gramínea + leguminosa) foram afetados $(\mathrm{P}<0,05)$ pela adubação fosfatada e potássica, não sendo detectado efeito significativo $(\mathrm{P}>0,05)$ para a interação entre os dois nutrientes. Para a gramínea, os rendimentos de MSV foram ajustados ao modelo quadrático de regressão e os máximos valores estimados com a aplicação de 87,4; 98,6; 77,5 e 78,2 kg de $\mathrm{kg}$ de $\mathrm{P}_{2} \mathrm{O}_{5}$ ha $^{-1}$, respectivamente, para a aplicação de $0,40,80$ e $120 \mathrm{~kg}$ de $\mathrm{kg}$ de $\mathrm{K}_{2} \mathrm{O}$ ha $^{-1}$, enquanto que para a soma dos componentes os maiores rendimentos de MSV foram obtidos com a aplicação de 115,7; 117,9; 102,3 e $90,3 \mathrm{~kg}$ de $\mathrm{kg}$ de $\mathrm{P}_{2} \mathrm{O}_{5} \quad \mathrm{ha}^{-1}$, respectivamente para a aplicação de $0,40,80$ e $120 \mathrm{~kg}$ de $\mathrm{kg}$ de $\mathrm{K}_{2} \mathrm{O}$ ha $^{-1}$. Na média dos níveis de adubação fosfatada e potássica, os maiores rendimentos de MSV da gramínea e da soma dos componentes foram obtidos com a aplicação de 73,8 e $105,1 \mathrm{~kg}^{\mathrm{de}} \mathrm{K}_{2} \mathrm{O} \mathrm{ha}^{-1}$ e 84,5 e $108,5 \mathrm{~kg}$ de $\mathrm{P}_{2} \mathrm{O}_{5} \mathrm{ha}^{-1}$, respectivamente. Os rendimentos de MSV da leguminosa foram diretamente proporcionais aos níveis de $\mathrm{P}$ e $\mathrm{K}$ (Tabela 1).

Costa, (2004), avaliando métodos de sobressemeadura de Desmodium ovalifolium em pastagens de Paspalum atratum cv. Pojuca, constatou que a adubação fosfatada e potássica foram indispensáveis para o pleno estabelecimento da leguminosa e a aplicação de 80 $\mathrm{kg}$ de $\mathrm{P}_{2} \mathrm{O}_{5}$ ha $^{-1}$, conjuntamente com $60 \mathrm{~kg}$ de $\mathrm{K}_{2} \mathrm{O}$ $\mathrm{ha}^{-1}$ foram a dose de máxima eficiência técnica, proporcionando maiores rendimentos de forragem da gramínea (4.876 $\mathrm{kg} \mathrm{ha}^{-1}$ de MSV), da leguminosa (2.371 $\mathrm{ha}^{-1}$ de MSV) e da consorciação (7.247 kg ha ${ }^{-1}$ de MSV). A participação da leguminosa na composição botânica da pastagem foi diretamente proporcional aos níveis de $\mathrm{P}(36,1 ; 35,7 ; 36,3$ e $40,5 \%$, respectivamente para $0,40,80$ e $120 \mathrm{~kg}$ de $\left.\mathrm{P}_{2} \mathrm{O}_{5} \mathrm{ha}^{-1}\right)$ e aos de $\mathrm{K}(33,2 ; 35,9 ; 36,8$ e $44,1 \%$, respectivamente para $0,40,80$ e $120 \mathrm{~kg}$ de $\mathrm{K}_{2} \mathrm{O}$ $\left.\mathrm{ha}^{-1}\right)$.

A estabilidade da composição botânica é o fator determinante no manejo de pastagens consorciadas, notadamente quanto a participação e persistência da leguminosa, considerada como componente mais valioso e instável da consorciação (Andrade et al., 2003, Townsend et al., 2012). Em regiões tropicais, a proporção adequada de leguminosas em pastagens consorciadas está na faixa de 20 a $40 \%$ da disponibilidade total de forragem, visando potencializar os benefícios decorrentes de sua presença, em termos de fixação biológica de $\mathrm{N}$ e maior concentração de nutrientes da forragem em oferta (Thomas, 1992, Ribeiro et al., 2007). A persistência da leguminosa depende diretamente do sistema e da pressão de pastejo utilizadas. Em geral, o pastejo contínuo e o alternado favorecem a permanência das leguminosas e são recomendados como ferramentas de manejo para assegurar sua manutenção na pastagem, considerando-se as variações estacionais da oferta de forragem, notadamente durante o período seco, desde que sejam utilizadas pressões de pastejo compatíveis e que assegurem a estabilidade produtiva da consorciação (Townsend et al., 2012, Costa et al., 2013b). 
Tabela 1. Rendimentos de matéria seca verde $\left(\mathrm{kg} \mathrm{ha}^{-1}\right)$ da gramínea Trachypogon plumosus, da leguminosa Stylosanthes capitata cv. Lavradeiro e da soma dos componentes (gramínea + leguminosa), em função da adubação fosfatada e potássica. Médias de onze cortes

\begin{tabular}{|c|c|c|c|c|c|}
\hline \multirow{2}{*}{$\begin{array}{l}\text { Doses de } \\
\mathrm{P}_{2} \mathrm{O}_{5} \mathrm{ha}^{-1} \\
\end{array}$} & \multicolumn{4}{|c|}{ Doses de $\mathrm{K}_{2} \mathrm{O}$ ha $^{-1}$} & \multirow{2}{*}{ Equações de Regressão } \\
\hline & 0 & 40 & 80 & 120 & \\
\hline & \multicolumn{5}{|c|}{ Gramínea } \\
\hline 0 & 2.132 & 2.598 & 2.981 & 2.544 & $Y=2.109+24,516 X-0,1402 X^{2}\left(R^{2}=0,97\right)$ \\
\hline 40 & 2.799 & 2.977 & 3.452 & 3.111 & $Y=2.546+18,307 X-0,0928 X^{2}\left(R^{2}=0,91\right)$ \\
\hline 80 & 3.241 & 3.571 & 3.789 & 3.388 & $Y=2.954+19,491 X-0,1258 X^{2}\left(R^{2}=0,88\right)$ \\
\hline \multirow[t]{2}{*}{120} & 3.011 & 3.356 & 3.455 & 3.099 & $\mathrm{Y}=2.530+20,905 \mathrm{X}-0,1338 \mathrm{X}^{2}\left(\mathrm{R}^{2}=0,93\right)$ \\
\hline & \multicolumn{5}{|c|}{ Leguminosa } \\
\hline 0 & 876 & 1.256 & 1.772 & 2.017 & $\mathrm{Y}=887,8+8,571 \mathrm{X}\left(\mathrm{R}^{2}=0,90\right)$ \\
\hline 40 & 1.245 & 1.558 & 1.875 & 2.218 & $\mathrm{Y}=1.261+6,835 \mathrm{X}\left(\mathrm{R}^{2}=0,93\right)$ \\
\hline 80 & 1.578 & 1.799 & 1.951 & 2.388 & $\mathrm{Y}=1.736+3,731 \mathrm{X}\left(\mathrm{R}^{2}=0,88\right)$ \\
\hline \multirow[t]{2}{*}{120} & 1.909 & 2.087 & 2.244 & 2.502 & $\mathrm{Y}=2.037+4,062 \mathrm{X}\left(\mathrm{R}^{2}=0,94\right)$ \\
\hline & \multicolumn{5}{|c|}{ Gramínea + Leguminosa } \\
\hline 0 & 3.008 & 3.854 & 4.753 & 4.561 & $\mathrm{Y}=2.987+33,809 \mathrm{X}-0,1461 \mathrm{X}^{2}\left(\mathrm{R}^{2}=0,91\right)$ \\
\hline 40 & 4.044 & 4.535 & 5.327 & 5.329 & $Y=3.808+22,405 X-0,0952 X^{2}\left(R^{2}=0,94\right)$ \\
\hline 80 & 4.819 & 5.370 & 5.740 & 5.776 & $Y=4.738+19,659 X-0,0961 X^{2}\left(R^{2}=0,95\right)$ \\
\hline 120 & 4.920 & 5.443 & 5.699 & 5.601 & $Y=4.546+26,599 X-0,1473 X^{2}\left(R^{2}=0,88\right)$ \\
\hline
\end{tabular}

Observou-se uma relação inversa entre os componentes da consorciação, pois à medida que os rendimentos de MSV da leguminosa foram incrementados, os da gramínea foram reduzidos, evidenciando os efeitos de competição interespecífica. As leguminosas forrageiras, em decorrência da menor habilidade na absorção de $\mathrm{P}$ e $\mathrm{K}$ que as gramíneas, apresentam maior responsividade à sua aplicação, refletindo os efeitos positivos sobre a nodulação e a fixação biológica do nitrogênio. Tendência semelhante foi repostada por Paciullo et al. (2002) para pastagens de Urochloa decumbens, cuja disponibilidade de forragem foi inversamente proporcional à de Stylosanthes guianensis cv. Mineirão. No entanto, Andrade et al. (2003) avaliando a mesma leguminosa, constataram a inviabilidade de sua consorciação com $U$. decumbens e Urochloa brizantha cv. Marandu, como consequência de sua pequena contribuição para a disponibilidade total de forragem (6,3 e 2,3\%, respectivamente), apesar da adequada disponibilidade de $\mathrm{K}$ no solo (75 mg $\mathrm{kg}^{-1}$ ).

Os teores de $\mathrm{N}, \mathrm{P}, \mathrm{Ca}, \mathrm{Mg}$ e $\mathrm{K}$ da gramínea foram ajustados ao modelo quadrático de regressão e os máximos valores estimados com a aplicação de 69,$8 ; 85,7 ; 82,0 ; 110$ e $83,2 \mathrm{~kg}$ de $\mathrm{P}_{2} \mathrm{O}_{5}$ ha $^{-1}$ e 86,7; 81,5; 63,0; 115,5 e 71,1 kg de $\mathrm{K}_{2} \mathrm{O}$ ha $^{-1}$, respectivamente. Para a leguminosa o efeito da adubação fosfatada sobre os teores de $\mathrm{N}$ foi positivo e linear, enquanto que as maiores concentrações de $\mathrm{P}, \mathrm{Ca}, \mathrm{Mg}$ e $\mathrm{K}$ foram estimadas com a aplicação de 96,$0 ; 102,1 ; 67,2$ e 94,4 kg de $\mathrm{P}_{2} \mathrm{O}_{5}$ ha $^{-1}$ e 60,5; 75,1; 70,2 e 71,0 kg de $\mathrm{K}_{2} \mathrm{O} \mathrm{ha}^{-1}$, respectivamente. (Tabelas 2 e 3 ). Com o desenvolvimento do dossel da pastagem o decréscimo na concentração de nutrientes, em função do maior acúmulo de forragem decorrente da adubação fosfatada, ocorre como consequência de dois fatores: 1) decréscimo na razão de área foliar (relação entre área foliar e a biomassa da planta), face ao maior investimento dos nutrientes na produção de biomassa estrutural e que não apresentam função fotossintetizante, visando promover o aumento em altura e tornar as plantas mais competitivas na captação da radiação incidente e; 2) decréscimo na concentração de nutrientes por unidade de área foliar das folhas sombreadas na porção inferior da planta, de modo a priorizar a alocação de nutrientes nas folhas superiores que apresentam maior taxa de fotossíntese (Lemaire and Agnusdei, 2000, Lemaire et al., 2011). No primeiro caso, a concentração de nutrientes nas plantas é uma função potencial negativa relacionada ao maior acúmulo de biomassa, notadamente durante o período vegetativo, sendo acentuada com baixa disponibilidade de nutrientes no solo, o qual não suprirá de forma satisfatória os requerimentos nutricionais da planta. No segundo caso, quando há competição entre plantas, um dos mecanismos para aumentar a interceptação da radiação incidente é o aumento da estatura da planta, a qual deve investir nutrientes, notadamente o $\mathrm{N}$, para a 
produção de colmos, estruturas que apresentam baixa concentração de nutrientes (Lemaire et al., 2008). Em pastagens de U. brizantha cv. Marandu consorciadas com Desmodium ovalifolium CIAT350, Costa (2004) constatou maiores concentrações de $\mathrm{N}, \mathrm{Ca}, \mathrm{Mg}$ e $\mathrm{K}$ da gramínea e leguminosa com a aplicação de doses entre 60 e 80 $\mathrm{kg}$ de $\mathrm{P}_{2} \mathrm{O}_{5} \mathrm{ha}^{-1}$ conjuntamente com 40 e $60 \mathrm{~kg}$ de $\mathrm{K}_{2} \mathrm{O}$ ha $^{-1}$, enquanto que os teores de $\mathrm{P}$ foram diretamente proporcionais às doses aplicadas $(0$, $30,60,90$ e $120 \mathrm{~kg}$ de $\left.\mathrm{P}_{2} \mathrm{O}_{5} \mathrm{ha}^{-1}\right)$.

Tabela 2. Teores $\left(\mathrm{g} \mathrm{kg}^{-1}\right)$ de nitrogênio $(\mathrm{N})$, fósforo $(\mathrm{P})$, cálcio $(\mathrm{Ca})$, magnésio $(\mathrm{Mg})$ e potássio $(\mathrm{K})$ da gramínea Trachypogon plumosus e da leguminosa Stylosanthes capitata cv. Lavradeiro, em função da adubação fosfatada e potássica.

\begin{tabular}{|c|c|c|c|c|c|c|c|c|c|}
\hline \multirow{3}{*}{ Variáveis } & \multirow{2}{*}{$\begin{array}{l}\text { Doses de } \\
\mathrm{P}_{2} \mathrm{O}_{5} \mathrm{ha}^{-1}\end{array}$} & \multicolumn{4}{|c|}{ Doses de $\mathrm{K}_{2} \mathrm{O}$ ha $^{-1}$} & \multicolumn{4}{|c|}{ Doses de $\mathrm{K}_{2} \mathrm{O}$ ha $^{-1}$} \\
\hline & & 0 & 40 & 80 & 120 & 0 & 40 & 80 & 120 \\
\hline & & \multicolumn{4}{|c|}{ Gramínea } & \multicolumn{4}{|c|}{ Leguminosa } \\
\hline \multirow{4}{*}{ Nitrogênio } & 0 & 12,21 & 12,98 & 13,78 & 12,55 & 25,33 & 27,57 & 29,77 & 28,21 \\
\hline & 40 & 12,97 & 13,04 & 14,78 & 13,66 & 26,93 & 28,28 & 29,66 & 27,51 \\
\hline & 80 & 13,21 & 13,98 & 14,91 & 14,27 & 28,56 & 30,02 & 31,46 & 29,22 \\
\hline & 120 & 13,02 & 13,62 & 14,33 & 14,02 & 29,11 & 30,86 & 31,87 & 30,57 \\
\hline \multirow{4}{*}{ Fósforo } & 0 & 1,03 & 1,34 & 1,47 & 1,33 & 1,39 & 1,52 & 1,61 & 1,54 \\
\hline & 40 & 1,28 & 1,49 & 1,66 & 1,59 & 1,62 & 1,74 & 1,66 & 1,59 \\
\hline & 80 & 1,41 & 1,56 & 1,78 & 1,67 & 1,78 & 1,91 & 1,97 & 1,87 \\
\hline & 120 & 1,37 & 1,51 & 1,72 & 1,61 & 1,71 & 1,83 & 1,89 & 1,70 \\
\hline \multirow{4}{*}{ Cálcio } & 0 & 3,97 & 4,59 & 5,31 & 4,88 & 5,82 & 6,39 & 6,98 & 6,41 \\
\hline & 40 & 4,56 & 4,88 & 5,65 & 5,21 & 6,44 & 6,77 & 7,02 & 6,56 \\
\hline & 80 & 4,98 & 5,17 & 5,88 & 5,44 & 6,98 & 7,88 & 8,15 & 7,21 \\
\hline & 120 & 4,33 & 5,31 & 5,63 & 5,02 & 6,17 & 7,11 & 8,01 & 7,49 \\
\hline \multirow{4}{*}{ Magnésio } & 0 & 1,81 & 1,97 & 2,33 & 2,07 & 2,98 & 3,59 & 4,45 & 3,81 \\
\hline & 40 & 2,35 & 2,98 & 3,02 & 2,85 & 3,17 & 3,98 & 4,72 & 4,11 \\
\hline & 80 & 2,89 & 3,33 & 3,81 & 3,22 & 3,89 & 4,36 & 4,88 & 4,33 \\
\hline & 120 & 2,37 & 3,51 & 4,01 & 3,64 & 3,44 & 4,01 & 4,65 & 4,59 \\
\hline \multirow{4}{*}{ Potássio } & 0 & 9,35 & 10,11 & 12,45 & 11,08 & 18,22 & 19,59 & 20,66 & 19,21 \\
\hline & 40 & 9,98 & 12,23 & 13,75 & 13,75 & 19,67 & 20,77 & 22,99 & 21,72 \\
\hline & 80 & 11,78 & 14,59 & 14,89 & 14,04 & 21,33 & 23,54 & 23,78 & 22,81 \\
\hline & 120 & 11,23 & 13,77 & 14,45 & 13,51 & 20,05 & 22,07 & 23,52 & 22,01 \\
\hline
\end{tabular}

Tabela 3. Equações de regressão ajustadas para os teores $\left(\mathrm{g} \mathrm{kg}^{-1}\right)$ de nitrogênio $(\mathrm{N})$, fósforo $(\mathrm{P})$, cálcio $(\mathrm{Ca})$, magnésio $(\mathrm{Mg})$ e potássio (K) da gramínea Trachypogon plumosus e da leguminosa Stylosanthes capitata cv. Lavradeiro, em função da adubação fosfatada e potássica.

\begin{tabular}{|c|c|c|}
\hline Variáveis & Gramínea & Leguminosa \\
\hline & \multicolumn{2}{|c|}{ Adubação Fosfatada } \\
\hline $\mathrm{N}$ & $Y=12,8+0,0279 X-0,00021 X^{2}\left(R^{2}=0,95\right)$ & $\mathrm{Y}=27,5+0,0259 \mathrm{X}\left(\mathrm{R}^{2}=0,91\right)$ \\
\hline $\mathrm{P}$ & $Y=1,3+0,00719 X-0,00004 X^{2}\left(R^{2}=0,91\right)$ & $Y=1,5+0,00069 X-0,000036 X^{2}\left(R^{2}=0,95\right)$ \\
\hline $\mathrm{Ca}$ & $\mathrm{Y}=4,7+0,0164 \mathrm{X}-0,00012 \mathrm{X}^{2}\left(\mathrm{R}^{2}=0,93\right)$ & $\mathrm{Y}=6,3+0,02042 \mathrm{X}-0,00011 \mathrm{X}^{2}\left(\mathrm{R}^{2}=0,88\right)$ \\
\hline $\mathrm{Mg}$ & $\mathrm{Y}=2,1+0,0242 \mathrm{X}-0,00041 \mathrm{X}^{2}\left(\mathrm{R}^{2}=0,89\right)$ & $\mathrm{Y}=3,6+0,01341 \mathrm{X}-0,00015 \mathrm{X}^{2}\left(\mathrm{R}^{2}=0,93\right)$ \\
\hline \multirow[t]{2}{*}{$\mathrm{K}$} & $\underline{Y}=10,7+0,0666 X-0,00043 X^{2}\left(R^{2}=0,95\right)$ & $Y=19,3+0,07552 X-0,00041 X^{2}\left(R^{2}=0,93\right)$ \\
\hline & \multicolumn{2}{|c|}{ Adubação Potássica } \\
\hline $\mathrm{N}$ & $Y=11,9+0,0347 X-0,00213 X^{2}\left(R^{2}=0,91\right)$ & $Y=27,3+0,0799 X-0,000542 X^{2}\left(R^{2}=0,93\right)$ \\
\hline $\mathrm{P}$ & $Y=1,2+0,00817 X-0,000051 X^{2}\left(R^{2}=0,93\right)$ & $Y=1,6+0,00484 X-0,000042 X^{2}\left(R^{2}=0,95\right)$ \\
\hline $\mathrm{Ca}$ & $\mathrm{Y}=4,4+0,0252 \mathrm{X}-0,00021 \mathrm{X}^{2}\left(\mathrm{R}^{2}=0,96\right)$ & $\mathrm{Y}=6,3+0,03101 \mathrm{X}-0,000022 \mathrm{X}^{2}\left(\mathrm{R}^{2}=0,93\right)$ \\
\hline $\mathrm{Mg}$ & $\mathrm{Y}=2,3+0,0231 \mathrm{X}-0,00013 \mathrm{X}^{2}\left(\mathrm{R}^{2}=0,92\right)$ & $\mathrm{Y}=3,3+0,02811 \mathrm{X}-0,000201 \mathrm{X}^{2}\left(\mathrm{R}^{2}=0,90\right)$ \\
\hline $\mathrm{K}$ & $Y=10,5+0,0786 X-0,00052 X^{2}\left(R^{2}=0,88\right)$ & $Y=19,7+0,0712 X-0,000561 X^{2}\left(R^{2}=0,96\right)$ \\
\hline
\end{tabular}


A gramínea e a leguminosa atenderiam, satisfatoriamente, aos requerimentos mínimos dos ruminantes em $\mathrm{N}$, independentemente dos níveis de adubação fosfatada ou potássica, considerandose que teores menores que $11,2 \mathrm{~g} \mathrm{~kg}^{-1}$ de MS são limitantes para uma adequada fermentação ruminal, implicando em menor consumo voluntário, redução na digestibilidade da forragem e balanço nitrogenado negativo (Minson, 2012). Os teores de $\mathrm{Ca}, \mathrm{Mg}$ e $\mathrm{K}$, em todos níveis de adubação fosfatada e potássica foram superiores ao nível crítico para bovinos de corte em crescimento $\left(1,8 ; \quad 1,0\right.$ e $\quad 6,5 \quad \mathrm{~g} \quad \mathrm{~kg}^{-1}$, respectivamente) recomendado pelo NRC (2000); enquanto que para o $\mathrm{P}\left(1,8 \mathrm{~g} \mathrm{~kg}^{-1}\right)$, as exigências seriam atendidas apenas pela leguminosa com a aplicação de $80 \mathrm{~kg}$ de $\mathrm{P}_{2} \mathrm{O}_{5}$ ha $^{-1}$.

Os efeitos da adubação fosfatada e potássica sobre a extração de macronutrientes da gramínea foram ajustados ao modelo quadrático de regressão, sendo os maiores valores para o N, P, Ca, Mg e K, respectivamente, estimados com a aplicação de 82,$5 ; 85,0 ; 84,2,102,8$ e $72,2 \mathrm{~kg}$ de $\mathrm{P}_{2} \mathrm{O}_{5}$ ha $^{-1}$ e 72,$4 ; 80,872,8 ; 72,9$ e $75,6 \mathrm{~kg}$ de $\mathrm{K}_{2} \mathrm{O} \mathrm{ha}^{-1}$, enquanto que para a leguminosa os efeitos foram lineares e positivos (Tabela 4$)$.

Tabela 4. Extração $\left(\mathrm{kg} \mathrm{ha}^{-1}\right)$ de nitrogênio $(\mathrm{N})$, fósforo $(\mathrm{P})$, cálcio $(\mathrm{Ca})$, magnésio $(\mathrm{Mg})$ e potássio $(\mathrm{K})$ da gramínea Trachypogon plumosus e da leguminosa Stylosanthes capitata cv. Lavradeiro, em função da adubação fosfatada e potássica.

\begin{tabular}{|c|c|c|c|c|c|c|c|c|c|}
\hline \multirow{3}{*}{ Variáveis } & \multirow{3}{*}{$\begin{array}{l}\text { Doses de } \\
\mathrm{P}_{2} \mathrm{O}_{5} \mathrm{ha}^{-1}\end{array}$} & \multicolumn{4}{|c|}{ Doses de $\mathrm{K}_{2} \mathrm{O}$ ha $^{-1}$} & \multicolumn{4}{|c|}{ Doses de $\mathrm{K}_{2} \mathrm{O} \mathrm{ha}^{-1}$} \\
\hline & & 0 & 40 & 80 & 120 & 0 & 40 & 80 & 120 \\
\hline & & \multicolumn{4}{|c|}{ Gramínea } & \multicolumn{4}{|c|}{ Leguminosa } \\
\hline \multirow{4}{*}{ Nitrogênio } & 0 & 26,0 & 33,7 & 41,1 & 31,9 & 22,2 & 34,6 & 52,8 & 56,9 \\
\hline & 40 & 36,3 & 38,8 & 51,0 & 42,5 & 33,5 & 44,1 & 55,6 & 61,0 \\
\hline & 80 & 42,8 & 49,9 & 56,5 & 48,3 & 45,1 & 54,0 & 61,4 & 69,8 \\
\hline & 120 & 39,2 & 45,7 & 49,5 & 43,4 & 55,6 & 64,4 & 71,5 & 76,5 \\
\hline \multirow{4}{*}{ Fósforo } & 0 & 2,2 & 3,5 & 4,4 & 3,4 & 1,2 & 1,9 & 2,9 & 3,1 \\
\hline & 40 & 3,6 & 4,4 & 5,7 & 4,9 & 2,0 & 2,7 & 3,1 & 3,5 \\
\hline & 80 & 4,6 & 5,6 & 6,7 & 5,7 & 2,8 & 3,4 & 3,8 & 4,5 \\
\hline & 120 & 4,1 & 5,1 & 5,9 & 5,0 & 3,3 & 3,8 & 4,2 & 4,3 \\
\hline \multirow{4}{*}{ Cálcio } & 0 & 8,5 & 11,9 & 15,8 & 12,4 & 5,1 & 8,0 & 12,4 & 12,9 \\
\hline & 40 & 12,8 & 14,5 & 19,1 & 16,2 & 8,0 & 10,5 & 13,2 & 14,6 \\
\hline & 80 & 16,1 & 18,5 & 22,3 & 18,4 & 11,1 & 14,2 & 15,9 & 17,2 \\
\hline & 120 & 13,0 & 17,8 & 19,5 & 15,6 & 11,8 & 14,8 & 18,0 & 18,7 \\
\hline \multirow{4}{*}{ Magnésio } & 0 & 3,9 & 5,1 & 6,9 & 5,3 & 2,6 & 4,5 & 7,9 & 7,7 \\
\hline & 40 & 6,6 & 8,9 & 10,4 & 8,9 & 3,9 & 6,2 & 8,9 & 9,1 \\
\hline & 80 & 9,4 & 11,9 & 14,4 & 10,9 & 6,1 & 7,8 & 9,5 & 10,3 \\
\hline & 120 & 7,1 & 11,7 & 13,9 & 11,3 & 6,6 & 8,4 & 10,4 & 11,5 \\
\hline \multirow{4}{*}{ Potássio } & 0 & 19,9 & 26,3 & 37,1 & 28,2 & 16,0 & 24,6 & 36,6 & 38,7 \\
\hline & 40 & 27,9 & 36,4 & 49,2 & 42,8 & 24,5 & 32,4 & 43,1 & 48,2 \\
\hline & 80 & 38,2 & 52,1 & 56,4 & 47,6 & 33,7 & 42,3 & 46,4 & 54,5 \\
\hline & 120 & 33,8 & 46,2 & 49,9 & 41,9 & 38,3 & 46,1 & 52,8 & 55,1 \\
\hline
\end{tabular}

Os decréscimos na absorção de macronutrientes com o aumento da produtividade de forragem podem decorrer da diminuição da capacidade fotossintética líquida do dossel, em função do sombreamento mútuo das folhas e da maior taxa respiratória das plantas, com reflexos diretos e negativos sobre as taxas de crescimento, implicando em menor demanda de nutrientes para a o atendimento dos processos metabólicos da gramínea e leguminosa (Lemaire and Agnusdei, 2000, Costa et al., 2010, Otsubo et al., 2011).
Costa (2004) reportou que as extrações de N, P, $\mathrm{Ca}, \mathrm{Mg}$ e $\mathrm{K}$ foram inversamente proporcionais ao índice de área foliar e negativamente correlacionadas com a disponibilidade de forragem em pastagens consorciadas de Paspalum atratum cv. Pojuca e Pueraria phaseoloides CIAT-9900. Para assegurar a produtividade de biomassa primária líquida satisfatória de $T$. plumosus (9.500 kg de MS ha ${ }^{-1}$ ano $^{-1}$ ), Medina (1982) e López-Hernández et al. (2006) sugerem como adequadas extrações de $\mathrm{N}$ entre 90 e $113 \mathrm{~kg}$ 
de $\mathrm{N} \mathrm{ha}^{-1}$ ano $^{-1}$, valores superiores aos constatados neste trabalho. Para todos os macronutrientes, as quantidades extraídas foram superiores às reportadas por Heringer and Jacques (2002) para pastagens nativas do Rio Grande do Sul, sem queima e sem utilização da roçagem $(41,3 ; 4,5$; 4,$8 ; 4,1$ e $51,1 \mathrm{~kg} \mathrm{ha}^{-1}$, respectivamente para $\mathrm{N}, \mathrm{P}$, $\mathrm{Ca}, \mathrm{Mg}$ e K).

As extrações dos macronutrientes pela gramínea e leguminosa, independentemente da adubação fosfatada ou potássica, foram maiores para $\mathrm{N}$ e K, seguidas de $\mathrm{Ca}, \mathrm{Mg}$ e $\mathrm{P}$ (Tabela 5). Esses resultados demonstram a necessidade de reposição dos macronutrientes extraídos visando maximizar a eficiência da adubação fosfatada, com reflexos positivos e significativos na disponibilidade de forragem da consorciação. Da mesma forma, Costa (2004) reportou efeitos significativos da adubação fosfatada (40, 80 e 120 $\mathrm{kg}$ de $\mathrm{P}_{2} \mathrm{O}_{5}$ ha $^{-1}$ ano $^{-1}$ ) e potássica $(30$ e $60 \mathrm{~kg}$ de $\mathrm{K}_{2} \mathrm{O}_{\text {ha }^{-1}}$ ano $^{-1}$ ) sobre a extração de $\mathrm{N}, \mathrm{P}, \mathrm{Ca}, \mathrm{Mg}$ e $\mathrm{K}$ por pastagens consorciadas de Paspalum guenoarum FCAP-46 e Stylosanthes capitata cv. Capica.

Os teores de FDN e FDA da gramínea e leguminosa foram inversamente proporcionais aos níveis de adubação fosfatada, independentemente dos níveis de adubação potássica (Tabela 6). Tendência semelhante foi reportada por Costa (2004) para pastagens consorciadas de $P$. atratum cv. Pojuca com $P$. phaseoloides CIAT-9900, onde a aplicação de $80 \mathrm{~kg}$ de $\mathrm{P}_{2} \mathrm{O}_{5}$ ha $^{-1}$ e $40 \mathrm{~kg}$ de $\mathrm{K}_{2} \mathrm{O}$ $\mathrm{ha}^{-1}$ proporcionou decréscimos superiores a $10 \%$ nos teores de fibra da gramínea (77,2 vs. 70,2\% de FDN e 39,1 vs. $34,3 \%$ de FDA) e da leguminosa (67,8 vs. $61,3 \%$ de FDN e 35,3 vs. $31,3 \%$ de FDA).

Tabela 5. Equações de regressão ajustadas para a extração $\left(\mathrm{kg} \mathrm{ha}^{-1}\right)$ de nitrogênio $(\mathrm{N})$, fósforo $(\mathrm{P})$, cálcio $(\mathrm{Ca})$, magnésio $(\mathrm{Mg})$ e potássio (K) da gramínea Trachypogon plumosus e da leguminosa Stylosanthes capitata cv. Lavradeiro, em função da adubação potássica.

\begin{tabular}{ccc}
\hline Variáveis & \multicolumn{2}{c}{ Gramínea } \\
\hline & \multicolumn{2}{c}{ Adubação Fosfatada } \\
\cline { 2 - 3 } $\mathrm{N}$ & $\mathrm{Y}=32,7+0,3632 \mathrm{X}-0,00221 \mathrm{X}^{2}\left(\mathrm{R}^{2}=0,93\right)$ & $\mathrm{Y}=40,91+0,2128 \mathrm{X}\left(\mathrm{R}^{2}=0,93\right)$ \\
$\mathrm{P}$ & $\mathrm{Y}=3,29+0,0511 \mathrm{X}-0,00032 \mathrm{X}^{2}\left(\mathrm{R}^{2}=0,94\right)$ & $\mathrm{Y}=2,31+0,0141 \mathrm{X}\left(\mathrm{R}^{2}=0,95\right)$ \\
$\mathrm{Ca}$ & $\mathrm{Y}=11,9+0,1516 \mathrm{X}-0,00091 \mathrm{X}^{2}\left(\mathrm{R}^{2}=0,90\right)$ & $\mathrm{Y}=9,64+0,0542 \mathrm{X}\left(\mathrm{R}^{2}=0,89\right)$ \\
$\mathrm{Mg}$ & $\mathrm{Y}=5,13+0,1258 \mathrm{X}-0,00062 \mathrm{X}^{2}\left(\mathrm{R}^{2}=0,91\right)$ & $\mathrm{Y}=5,58+0,0301 \mathrm{X}\left(\mathrm{R}^{2}=0,91\right)$ \\
$\mathrm{K}$ & $\mathrm{Y}=27,2+0,4519 \mathrm{X}-0,00261 \mathrm{X}^{2}\left(\mathrm{R}^{2}=0,95\right)$ & $\mathrm{Y}=29,91+0,1612 \mathrm{X}\left(\mathrm{R}^{2}=0,95\right)$ \\
& & Adubação Potássica \\
$\mathrm{N}$ & $\mathrm{Y}=35,3+0,3187 \mathrm{X}-0,00219 \mathrm{X}^{2}\left(\mathrm{R}^{2}=0,89\right)$ & $\mathrm{Y}=39,92+0,2293 \mathrm{X}\left(\mathrm{R}^{2}=0,88\right)$ \\
$\mathrm{P}$ & $\mathrm{Y}=3,49+0,0485 \mathrm{X}-0,00035 \mathrm{X}^{2}\left(\mathrm{R}^{2}=0,90\right)$ & $\mathrm{Y}=2,41+0,0125 \mathrm{X}\left(\mathrm{R}^{2}=0,91\right)$ \\
$\mathrm{Ca}$ & $\mathrm{Y}=12,21+0,1601 \mathrm{X}-0,0011 \mathrm{X}^{2}\left(\mathrm{R}^{2}=0,94\right)$ & $\mathrm{Y}=9,43+0,0575 \mathrm{X}\left(\mathrm{R}^{2}=0,94\right)$ \\
$\mathrm{Mg}$ & $\mathrm{Y}=6,52+0,1167 \mathrm{X}-0,00081 \mathrm{X}^{2}\left(\mathrm{R}^{2}=0,93\right)$ & $\mathrm{Y}=5,02+0,0432 \mathrm{X}\left(\mathrm{R}^{2}=0,92\right)$ \\
$\mathrm{K}$ & $\mathrm{Y}=29,3+0,4389 \mathrm{X}-0,00292 \mathrm{X}^{2}\left(\mathrm{R}^{2}=0,92\right)$ & $\mathrm{Y}=28,84+0,1785 \mathrm{X}\left(\mathrm{R}^{2}=0,95\right)$ \\
\hline
\end{tabular}

Gama et al. (2013) constataram reduções nos teores de FDN $(68,1 \%)$ e FDA $(33,1 \%)$ de Urochloa decumbens cv. Basilisk em consórcio com Stylosanthes cv. Campo Grande, comparativamente ao cultivo isolado da gramínea (70,3\% de FDN e 34,9\% de FDA). Nas plantas forrageiras, a adubação ao estimular as taxas de aparecimento e alongamento de folhas, com reflexos positivos em seu comprimento final e na sua duração de vida, contribui para redução ou manutenção dos teores de fibra, apesar dos acréscimos nos níveis de produtividade de forragem (Benett et al., 2008, Costa et al., 2008, Costa et al., 2013a). Com o envelhecimento da gramínea a proporção dos componentes potencialmente digestíveis apresenta uma tendência a diminuir e a de tecidos fibrosos, aumentar, sendo o conteúdo da parece celular o fator mais limitante ao desempenho produtivo de ruminantes consumindo gramíneas forrageiras tropicais (Silva and Haridasan, 2007).

A FDN, constituída por celulose, hemicelulose, lignina e sílica, afeta diretamente o consumo voluntário, devido a maior taxa de enchimento e a menor taxa de passagem do alimento no sistema digestivo, enquanto que a FDA se correlaciona negativamente com a disponibilidade de energia e a digestibilidade da forragem (Norton, 1982). 
Tabela 6. Teores (\%) de fibra em detergente neutro (FDN) e fibra em detergente ácido (FDA) da gramínea Trachypogon plumosus e da leguminosa Stylosanthes capitata cv. Lavradeiro, em função da adubação fosfatada e potássica.

\begin{tabular}{|c|c|c|c|c|c|c|}
\hline \multirow{2}{*}{ Variáveis } & \multirow{2}{*}{$\begin{array}{l}\text { Doses de } \\
\mathrm{P}_{2} \mathrm{O}_{5} \mathrm{ha}^{-1}\end{array}$} & \multicolumn{4}{|c|}{ Doses de $\mathrm{K}_{2} \mathrm{O}$ ha $^{-1}$} & \multirow{2}{*}{ Equações de Regressão } \\
\hline & & 0 & 40 & 80 & 120 & \\
\hline \multirow{5}{*}{ FDN } & & \multicolumn{5}{|c|}{ Gramínea } \\
\hline & 0 & 76,12 & 74,98 & 74,11 & 73,77 & $Y=76,37-0,0416 X\left(R^{2}=0,89\right)$ \\
\hline & 40 & 75,01 & 73,91 & 73,05 & 72,84 & $\mathrm{Y}=75,17-0,0346 \mathrm{X}\left(\mathrm{R}^{2}=0,93\right)$ \\
\hline & 80 & 73,21 & 72,71 & 72,22 & 71,98 & $\mathrm{Y}=74,35-0,0342 \mathrm{X}\left(\mathrm{R}^{2}=0,96\right)$ \\
\hline & 120 & 71,17 & 70,76 & 69,83 & 69,21 & $\mathrm{Y}=74,13-0,0364 \mathrm{X}\left(\mathrm{R}^{2}=0,91\right)$ \\
\hline \multirow[t]{4}{*}{ FDA } & 0 & 39,65 & 38,88 & 37,21 & 37,07 & $\mathrm{Y}=39,82-0,0194 \mathrm{X}\left(\mathrm{R}^{2}=0,92\right)$ \\
\hline & 40 & 39,11 & 38,05 & 37,36 & 36,57 & $\mathrm{Y}=38,97-0,0224 \mathrm{X}\left(\mathrm{R}^{2}=0,96\right)$ \\
\hline & 80 & 38,66 & 37,50 & 36,44 & 35,99 & $Y=37,41-0,0127 X\left(R^{2}=0,85\right)$ \\
\hline & 120 & 37,21 & 36,08 & 35,82 & 35,04 & $\mathrm{Y}=37,17-0,0167 \mathrm{X}\left(\mathrm{R}^{2}=0,96\right)$ \\
\hline \multirow{5}{*}{ FDN } & & \multicolumn{5}{|c|}{ Leguminosa } \\
\hline & 0 & 65,87 & 64,79 & 64,12 & 63,96 & $\mathrm{Y}=65,91-0,0361 \mathrm{X}\left(\mathrm{R}^{2}=0,96\right)$ \\
\hline & 40 & 64,56 & 63,98 & 63,51 & 63,15 & $\mathrm{Y}=65,07-0,0373 \mathrm{X}\left(\mathrm{R}^{2}=0,94\right)$ \\
\hline & 80 & 62,98 & 62,11 & 61,37 & 60,89 & $\mathrm{Y}=64,64-0,0443 \mathrm{X}\left(\mathrm{R}^{2}=0,95\right)$ \\
\hline & 120 & 61,59 & 60,44 & 58,93 & 58,16 & $Y=64,48-0,0492 X\left(R^{2}=0,92\right)$ \\
\hline \multirow[t]{4}{*}{ FDA } & 0 & 37,36 & 36,98 & 36,45 & 35,99 & $\mathrm{Y}=37,45-0,0103 \mathrm{X}\left(\mathrm{R}^{2}=0,93\right)$ \\
\hline & 40 & 37,11 & 36,22 & 36,03 & 35,41 & $\mathrm{Y}=36,92-0,0136 \mathrm{X}\left(\mathrm{R}^{2}=0,97\right)$ \\
\hline & 80 & 36,81 & 35,98 & 35,27 & 35,01 & $\mathrm{Y}=36,50-0,0145 \mathrm{X}\left(\mathrm{R}^{2}=0,91\right)$ \\
\hline & 120 & 36,09 & 35,24 & 34,77 & 34,23 & $\mathrm{Y}=36,01-0,0142 \mathrm{X}\left(\mathrm{R}^{2}=0,90\right)$ \\
\hline
\end{tabular}

Para todos os níveis de adubação fosfatada e potássica, os teores de FDN e FDA da gramínea e da leguminosa, exceto para os teores de FDN da leguminosa fertilizada com $120 \mathrm{~kg}$ de $\mathrm{P}_{2} \mathrm{O}_{5} \mathrm{ha}^{-1} \mathrm{e}$ 80 ou $120 \mathrm{~kg}$ de $\mathrm{K}_{2} \mathrm{O} \mathrm{ha}^{-1}$, foram superiores aos limites sugeridos por Van Soest (1994), 30 e 60\% para FDA e FDN, respectivamente, como indicadores de forragem de alta qualidade, pois forragens com valores de FDA em torno de 40\%, ou mais, apresentam acentuada redução no consumo voluntário e na sua digestibilidade. Contudo, os teores foram inferiores aos relatados por Townsend et al. (2012) para Axonopus purpusii $(78,9 \%$ de FDN e $46,1 \%$ de FDA) e Mesosetum chaseae $(77,1 \%$ de FDN e 47,9\% de FDA), gramíneas nativas dos cerrados de Rondônia.

\section{Conclusões}

A adubação fosfatada e potássica afetam positivamente o rendimento de forragem, a composição química e a extração de macronutrientes pela gramínea e leguminosa.

As extrações dos macronutrientes pela gramínea e leguminosa, independentemente da adubação fosfatada ou potássica, são maiores para $\mathrm{N}$ e K, seguidas de $\mathrm{Ca}, \mathrm{Mg}$ e $\mathrm{P}$.

A adubação fosfatada e potássica proporcionam forragem da gramínea e leguminosa com melhor qualidade e menores teores de fibra.
Considerando-se a disponibilidade total de forragem e a composição botânica da pastagem, a utilização de níveis entre 80 a $90 \mathrm{~kg}$ de $\mathrm{P}_{2} \mathrm{O}_{5} \mathrm{ha}^{-1} \mathrm{e}$ 60 a $70 \mathrm{~kg}$ de $\mathrm{K}_{2} \mathrm{O} \quad \mathrm{ha}^{-1}$ possibilita o estabelecimento, a estabilidade produtiva, o fornecimento de forragem de boa qualidade e a persistência da consorciação.

\section{Referências Bibliográficas}

Andrade, C. M, Garcia, R., Couto, L., Pereira, O. G. \& Souza, A. 2003. Desempenho de seis gramíneas solteiras ou consorciadas com o Stylosanthes guianensis cv. Mineirão e eucalipto em sistema silvipastoril. Revista Brasileira de Zootecnia, 32, 1845-1850.

Benett, C. G. S., Yamashita, O. M., Koga, P. S. \& Silva, K. S. 2008. Resposta da Brachiaria brizantha cv. Marandu a diferentes tipos de adubação. Revista de Ciências AgroAmbientais, 6, 13-20.

Costa, K. A. P., Araujo, J. L., Faquin, V., Oliveira, I. P., Figueiredo, F. C. \& Gomes, K. W. 2008. Extração de macronutrientes pela fitomassa do capim-xaraés "xaraés" em função de doses de nitrogênio e potássio. Ciência Rural, 38, 11621166.

Costa, K. A. P., Oliveira, I. P., Severiano, E. C., Sampaio, F. d. M. T., Carrijo, M. S. \& Rodrigues, C. R. 2010. Extração de nutrientes pela fitomassa de cultivares de Brachiaria 
brizantha sob doses de nitrogênio. Ciência Animal Brasileira, 11, 307-314.

Costa, N. L. 2004. Formação, manejo $e$ recuperação de pastagens em Rondônia. Porto Velho: Embrapa Rondônia. 217p.

Costa, N. L., Gianluppi, V., Braga, R. M. \& Bendahan, A. B. 2009. Alternativas tecnológicas para a pecuária de Roraima. Boa Vista: Embrapa Roraima, 35p. (Documentos, 19).

Costa, N. L., Moraes, A., Carvalho, P. C. F., Monteiro, A. L. G., Motta, A. C. V. \& Oliveira, R. A. 2013a. Composição e extração de nutrientes por Trachypogon plumosus sob adubação e idades de rebrota. Archivos de Zootecnia, 62, 227-238.

Costa, N. L., Moraes, A., Monteiro, A. L. G., Motta, A. C. V., Oliveira, R. A. \& Rodrigues, A. N. A. 2013b. Forage productivity and morphogenesis of Axonopus aureus under different nitrogen fertilization rates. Revista Brasileira de Zootecnia, 42, 541-548.

Fabrice, C. E. S., Soares Filho, C. V., Pinto, M. F., Perri, S. H. V., Cecato, U. \& Mateus, G. P. 2015. Recuperação de pastagens de Brachiaria decumbens degradada com introdução de Stylosanthes e adubação fosfatada. Revista Brasileira de Saúde e Produção Animal, 16, 758-771.

Ferreira, D. F. 2011. SISVAR: A Computer Statistical Analysis System. Ciência $e$ Agrotecnologia, 35, 1039-1042.

Gama, T. C. M., Volpe, E., Lempp, B. \& Costa Galdeia, E. 2013. Recuperação de pasto de capim-braquiária com correção e adubação de solo e estabelecimento de leguminosas. Revista Brasileira de Saúde e Produção Animal, 14, 635-647.

Gianluppi, D., Gianluppi, V. \& Smiderle, O. J. 2001. Produção de pastagens nos cerrados de Roraima. Boa Vista: Embrapa Roraima, 4p. (Comunicado Técnico, 14).

Heringer, I. \& Jacques, A. V. A. 2002. Qualidade da forragem de pastagem nativa sob distintas alternativas de manejo. Pesquisa Agropecuária Brasileira, 37, 399-406.

Köppen, W. \& Geiger, R. 1928. Klimate der Erde. Gotha: Verlag Justus Perthes. Wall-map $150 \mathrm{~cm} \times 200 \mathrm{~cm}$.

Lemaire, G. \& Agnusdei, M. 2000. Leaf tissue turnover and efficiency of herbage utilization. In: Lemaire, G., Hodgson, J., Moraes, A.,
Carvalho, P. C. F. \& Nabinger, C. (Eds.) Grassland ecophysiology and grazing ecology. $\mathrm{CAB}$ International Publishing, New York. p.265-288.

Lemaire, G., Hodgson, J. \& Chabbi, A. 2011. Grassland productivity and ecosystem services. Cabi, Wallingford. 287p.

Lemaire, G., Jeuffroy, M.-H. \& Gastal, F. 2008. Diagnosis tool for plant and crop $\mathrm{N}$ status in vegetative stage: Theory and practices for crop $\mathrm{N}$ management. European Journal of Agronomy, 28, 614-624.

López-Hernández, D., Santaella, S. \& Chacón, P. 2006. Contribution of nitrogen-fixing organisms to the $\mathrm{N}$ budget in Trachypogon savannas. European Journal of Soil Biology, 42, 43-50.

Medina, E. 1982. Nitrogen balance in the Trachypogon grasslands of Central Venezuela. Plant and Soil, 67, 305-314.

Minson, D. 2012. Forage in ruminant nutrition. Academic Press, New York.

Moreira, A. L. 2006. Melhoramento de pastagens através da técnica da sobressemeadura de forrageiras de inverno. Pesquisa e Tecnologia, 3, 1-9.

Moreira, E. M. \& Arruda, N. G. 1990. Métodos de introdução de Pueraria phaseoloides em pastagens de Brachiaria decumbens. Reunión de la red internacional de evaluación de pastos tropicales-Amazonia, 1, 945-948.

Nabinger, C. \& Carvalho, P. C. F. 2009. Ecofisiología de sistemas pastoriles: aplicaciones para su sustentabilidad. Agrociencia, 13, 18-27.

Norton, B. W. 1982. Differences between species in forage quality. In: Hacker, J. B. (Ed.) Nutritional limits to animal production from pastures. Farnham Royal, Queensland, Australia. p.89-110.

NRC. 2000. Nutrient Requirements of Beef Cattle, 7th rev. edn. Natl. Acad. Press, Washington, DC, USA. 242p.

Otsubo, A. A., Brito, O. R., Schnitzer, J. A. \& Otsubo, V. H. N. 2011. Produção de matéria seca de Stylosanthes capitata submetido à adubação fosfatada em solos com diferentes texturas. Semina: Ciências Agrárias, 32, $1677-$ 1686.

Paciullo, D. S. C., Gomide, J. A., Silva, E. A. M., Queiroz, D. S. \& Gomide, C. A. M. 2002. Características anatômicas da lâmina foliar e 
do colmo de gramíneas forrageiras tropicais, em função do nível de inserção no perfilho, da idade e da estação de crescimento. Revista Brasileira de Zootecnia, 31, 890-899.

Ribeiro, R. C., Rossiello, R. P., Macedo, R. O. \& Barbieri Júnior, E. B. 2007. Introdução de desmódio em pastagem estabelecida de Brachiaria humidicola: densidade e frequência da leguminosa no consórcio. Revista da Universidade Rural, 27, 41-49.

Ries, L. P. \& Shugart, H. H. 2008. Nutrient limitations on understory grass productivity and carbon assimilation in an African woodland savanna. Journal of Arid Environments, 72, 1423-1430.

Sarmiento, G. 1992. Adaptive strategies of perennial grasses in South American savannas. Journal of Vegetation Science, 3, 325-336.

Sarmiento, G., Silva, M. P., Naranjo, M. E. \& Pinillos, M. 2006. Nitrogen and phosphorus as limiting factors for growth and primary production in a flooded savanna in the Venezuelan Llanos. Journal of Tropical Ecology, 22, 203-212.

Silva, D. J. \& Queiroz, A. C. 2002. Análise de alimentos: métodos químicos e biológicos. 3.Ed. Universdiade Federal de Viçosa, Viçosa, Minas Gerais, Brasil. 305p.

Silva, F. C. 2009. Manual de análises químicas de solos, plantas e fertilizantes. Embrapa Informação Tecnológica, Rio de Janeiro. 370p.
Silva, J. S. O. \& Haridasan, M. 2007. Acúmulo de biomassa aérea e concentração de nutrientes em Melinis minutiflora P. Beauv. e gramíneas nativas do cerrado. Revista Brasileira de Botânica, 30, 337-344.

Thomas, R. J. 1992. The role of the legume in the nitrogen cycle of productive and sustainable pastures. Grass and Forage Science, 47, 133142.

Townsend, C. R., Costa, N. L. \& Pereira, R. G. A. 2012. Pastagens nativas da amazônia brasileira. Porto Velho: Embrapa Rondônia. 30p. (Documentos, 149).

Van Soest, P. J. 1994. Nutritional ecology of the ruminant. Cornell University Press, Ithaca, NY, USA. 476p.

Van Soest, P. J., Robertson, J. B. \& Lewis, B. A. 1991. Methods for dietary fiber, neutral detergent fiber, and nonstarch polysaccharides in relation to animal nutrition. Journal of Dairy Science, 74, 3583-3597.

Article History:

Received 7 June 2017

Accepted 7 August 2017

Available online 28 August 2017

License information: This is an open-access article distributed under the terms of the Creative Commons Attribution License 4.0, which permits unrestricted use, distribution, and reproduction in any medium, provided the original work is properly cited. 